\title{
Provider Experiences with a Payer-Based PCMH Program
}

\author{
Gilbert Gimm, $P h D^{7}$, Debora G. Goldberg, $P h D^{2}$, Nouran Ghanem, MSHP2, \\ Sahar Haghighat, $M A^{2}$, Jay Want, $M^{3}$, Dan Hough, $M B A^{4}$, and Len M. Nichols, PhD ${ }^{2}$ \\ 'Department of Health Administration and Policy, George Mason University, Fairfax, VA, USA; ${ }^{2}$ George Mason University, Fairfax, VA, USA; ${ }^{3}$ Peterson \\ Center on Healthcare, New York, NY, USA; ${ }^{4}$ Alan Newman Research, Richmond, VA, USA.
}

\begin{abstract}
BACKGROUND: The patient-centered medical home $(\mathrm{PCMH})$ is an enhanced primary care model that aims to improve quality of care. Over the past several years, the PCMH model has been adopted by Medicare and private payers, which offer financial resources and technical assistance to participating practices. However, few studies have examined provider experiences and perspectives on the adoption of payer-based PCMH models in different practice settings.
\end{abstract}

OBJECTIVE: The goal of this qualitative study was to analyze how providers experienced specific elements of a payer-based PCMH model and identify cross-cutting themes that can be applied to other payer-based PCMH initiatives.

DESIGN: Observational qualitative study.

PARTICIPANTS: A total of 65 individuals (which includes 57 primary care physicians) participated in focus group sessions. Telephone interviews were conducted with an additional 14 physicians and 2 practice administrators.

APPROACH: Interviews and focus groups were recorded after obtaining the informed consent of participants. Written transcripts from the recordings were then imported into NVivo 11 for subsequent coding and qualitative analysis of themes.

KEY RESULTS: We found that nurse care coordinators (NCCs) were the single most valuable and visible program element. Individual care plans served as effective tools of communication between the NCC and physician on patient care management goals and issues. The online data portal was viewed as the least valuable element. With regard to cross-cutting themes, some providers expressed a strained relationship with CareFirst due to communication challenges, a lack of trust, and differing priorities in selecting patients for care plans.

CONCLUSION: Nurse care coordinators and the targeted use of individualized care plans are essential components in a payer-based PCMH program. Improving communication and trust in data reports are critical for effective implementation. Future research should examine provider experiences in other payer-based PCMH programs to see how interactions with payers affect program implementation.

Electronic supplementary material The online version of this article (https://doi.org/10.1007/s11606-019-05005-7) contains supplementary material, which is available to authorized users.

Received September 12, 2018

Revised January 17, 2019

Accepted March 14, 2019

Published online April 22, 2019
KEY WORDS: provider experiences; qualitative; patient-centered medical home.

J Gen Intern Med 34(10):2047-53

DOI: $10.1007 / \mathrm{s} 11606-019-05005-7$

(c) Society of General Internal Medicine 2019

\section{INTRODUCTION}

The patient-centered medical home (PCMH) is an enhanced primary care model that aims to improve quality of care. Since 2007, the joint principles of a PCMH have included a personal physician, whole person orientation, coordinated care, quality and safety, and enhanced access. ${ }^{1}$ A key element in PCMH models is care coordination, which typically includes a nurse care coordinator (NCC) who identifies patients with complex needs, conducts follow-up calls with patients after visits, and monitors their status. ${ }^{2,3}$

Over the past several years, the PCMH model has been adopted by Medicare and private payers, which offer financial resources and technical assistance to practices that agree to participate. ${ }^{4}$ However, full implementation of PCMH models can be challenging due to resource and time constraints, data and infrastructure limitations, and the need to change existing workflows. ${ }^{5}$ Also, the cost of external PCMH certification by the National Committee for Quality Assurance (NCQA) may be a barrier for small practices. ${ }^{6}$ As a result, small primary practices with one or two physicians have adopted relatively few PCMH processes. ${ }^{7,8}$

The majority of PCMH evaluations have focused on cost, quality, or utilization outcomes. ${ }^{9-14}$ However, few studies have examined provider experiences and perspectives on the adoption of payer-based PCMH models in different practice settings. ${ }^{6,15}$ Provider perspectives can help to elucidate our understanding of which PCMH program components are effective or need further refinement. To address this information gap, this qualitative study analyzed how providers experienced specific elements of a payer-based PCMH model and identified several cross-cutting themes that can be applied to other payer-based PCMH initiatives.

\section{PCMH Program Description and Elements}

In 2011, CareFirst BlueCross BlueShield of Maryland implemented a voluntary PCMH program, but did not require 
practices to obtain external NCQA certification or make internal investments in staffing resources or electronic medical record (EMR) systems. The goal of the CareFirst PCMH program was to improve the health of CareFirst members and control growth in health care costs. CareFirst developed and grouped all participating primary care physicians and nurse practitioners into organizational units called Medical Care Panels, or "Panels," to create a larger patient population base to track quality and cost savings as well as calculate incentive payments. The panels were also designed to encourage peer consultation across practices as well as competition within and across panels.

Although the CareFirst PCMH model included many features, five elements comprised the core of the program. First, CareFirst offered financial incentives with a $12 \%$ reimbursement increase for all participating groups that agreed to join the PCMH program. Also, participants were eligible for larger reimbursement increases, called outcome incentive awards (OIAs), if their annual total cost of care and quality scores met a performance target. Second, external nurse care coordinators (NCCs) were provided at no charge to practices. The NCCs helped to manage high-cost patients and were highly visible because of their supportive interactions with clinicians and office staff.

A third program element was the use of individualized care plans for a select group of patients. The NCC worked together with providers to develop care plans, which included a patient narrative, social and family history, medications, allergies, vital signs, encounter history, assessment, and care coordination team information. The care plan, however, was kept separate from the practice's own information system and not used to replace existing medical records. ${ }^{16}$ Rather, it was an additional document that tracked notes on a patient's condition and health-related needs based on the collaborative input of the NCC and primary care provider (PCP).

A fourth element was the quarterly panel meeting, in which CareFirst representatives gave a summary of PCMH program updates and performance reports, which indicated a panel's total cost of care and quality scores. The fifth element was a secure data portal, called Searchlight, which gave participating providers direct access to information on total cost of care, including primary care visits, specialist visits, and hospitalizations, for the 50 highest-cost patient members in a panel. However, the Searchlight data portal required an additional, multi-faceted login process that was separate from a practice's existing information systems.

\section{METHODS}

The goals of this study were to understand provider perceptions of the CareFirst PCMH program, examine provider experiences with key program elements, and identify suggestions for program improvement. To meet these objectives, we conducted focus groups and telephone interviews with participating providers and practice administrators in the CareFirst PCMH program. All research protocols, focus group moderator guides, and data collection instruments were approved by the George Mason University Institutional Review Board.

\section{Focus Group Participants}

We used a maximum variation approach ${ }^{17}$ for sampling that allowed us to recruit different types of panels with varying numbers of CareFirst care plans. Our team recruited eight panels that had participated in focus groups in a previous study of the CareFirst PCMH program. ${ }^{6}$ Following up with the same group of providers allowed us to examine changes in their perceptions and use of program elements after several years of experience with PCMH program implementation. Panels in this study represented primary care practices focused on adult patients, with each panel comprised of 7 to 15 providers. Virtual panels were clusters of small independently owned practices with 1 or 2 PCPs. Single independent panels consisted of a group of 10 to 15 PCPs from the same independently owned practice. Health system panels were clusters of practices owned by a large corporate entity. Table 1 includes characteristics of the eight panels studied.

\section{Data Collection}

Focus groups and telephone interviews were conducted between May 2016 and November 2017 with PCMH physicians, nurse practitioners, and office administrators. A total of 65 individuals participated in focus group sessions, 57 of which were primary care physicians. Telephone interviews were conducted with an additional 14 physicians and 2 practice administrators when key individuals were unable to attend a focus group. Participants were compensated between $\$ 125$ and \$200 for study participation, depending on their role and level of involvement. Each participant gave informed consent for the interview and audio recording.

Table 1 Characteristics of Panels Participating in Focus Groups

\begin{tabular}{|c|c|c|c|c|c|}
\hline $\begin{array}{l}\text { Panel } \\
\text { number }\end{array}$ & $\begin{array}{l}\text { Panel } \\
\text { type }\end{array}$ & $\begin{array}{l}\text { Number } \\
\text { of PCPs }\end{array}$ & State & $\begin{array}{l}\text { Care } \\
\text { plans } \\
\text { per } \\
\text { year } \\
(\mathbf{2 0 1 4})\end{array}$ & $\begin{array}{l}\text { NCQA } \\
\text { PCMH } \\
\text { certified* }\end{array}$ \\
\hline 1 & Virtual & 11 & MD & 51 & No \\
\hline 2 & Virtual & 15 & MD & 49 & No \\
\hline 3 & $\begin{array}{l}\text { Single } \\
\text { independent }\end{array}$ & 10 & MD & 33 & No \\
\hline 4 & $\begin{array}{l}\text { Single } \\
\text { independent }\end{array}$ & 15 & VA & 24 & No \\
\hline 5 & $\begin{array}{l}\text { Health } \\
\text { system }\end{array}$ & 13 & MD & 53 & Yes \\
\hline 6 & $\begin{array}{l}\text { Health } \\
\text { system }\end{array}$ & 14 & VA & 7 & Yes \\
\hline 7 & $\begin{array}{l}\text { Health } \\
\text { system }\end{array}$ & 9 & MD & 15 & Yes \\
\hline 8 & $\begin{array}{l}\text { Health } \\
\text { system }\end{array}$ & 7 & MD & 7 & Yes \\
\hline
\end{tabular}

*NCQA recognition denotes PCMH certification of all practices within a panel 
All data collection efforts were directed by research protocols and a semi-structured interview guide developed by our internal research team. Experienced facilitators from Alan Newman Research, a consulting firm that specializes in physician research, conducted the focus groups and interviews. Facilitators used member checking throughout the interview process by rephrasing key statements back to participants to verify accurate interpretation of participant responses, which led to increased validity and credibility of the data. ${ }^{18}$ Members from our internal research team participated in 5 of the 8 focus group sessions.

\section{Data Analysis}

Qualitative analysis of the interview transcripts was performed by a multidisciplinary team of researchers to maximize coding insights. Our team consisted of specialists in health economics, primary care medicine, organization and management, practice transformation, and qualitative and interpretive sociology. Four members of our research team completed an initial review of 3 transcripts to produce a preliminary list of qualitative codes. We used this list as a codebook in NVivo (version 11), a qualitative data analysis software program, for coding all 24 transcripts. In addition to structured coding, we also used an "open coding" approach to identify potential themes that emerged from the data. The research team met on a regular basis to discuss codebook revisions and emerging themes.

At least two researchers independently reviewed and coded each transcript. We adhered to the conventional standard of kappa values greater than 0.4 represented good interrater reliability. ${ }^{19}$ When kappa values were below 0.4 , the research team met to reconcile differences and reach agreement before revising text coding. After this reconciliation process, the coding comparison queries for these transcripts were subsequently rerun with achievement of all kappa values greater than 0.4 .

\section{RESULTS}

\section{Provider Experiences: PCMH Program Elements}

Nurse Care Coordinators. Across the board, providers made explicit that the NCC was the single most valuable and visible program element. Providers expressed unanimous sentiment that the NCC was strongly linked to the overall efficacy of the CareFirst PCMH model. Focus groups with providers also revealed that over time, the NCCs became more integrated with care teams and trusted to assume greater responsibility for CareFirst PCMH tasks and patient care.

Physician feedback conveyed two ways by which NCCs added value. First, NCCs supported patients through care coordination activities such as making appointments for patients, holding weekly patient calls, and training patients with self-management skills. Second, the NCCs supported physicians by initiating the care plan process. The effectiveness of NCCs varied in terms of delivering patient care and adapting to a practice's workflow. Effective NCCs were thought to be proactive, engaged, and resolutely committed to patient care. Descriptors like "personality" and "assertiveness" were used many times to characterize an effective NCC.

It's a collaborative effort so physicians have to be committed to it, but at the same time, they have to give us NCCs who are equally as committed, equally as engaged with us.

Providers found that NCC follow-up communications with patients after a visit were particularly valuable. Many providers also stated that patients with care plans developed a close relationship with the NCC and were more likely to disclose non-compliance issues with the NCC as a result.

Some of my patients develop a trust with the NCC, they will tell them things they won't tell me because they're afraid that I'll be angry with them.

... If there are any questions, they actually call the NCC... they have a very close relationship. They feel comfortable working with them ...

In contrast, NCCs who were inflexible or unreceptive to provider input were perceived as less effective. Providers were unsettled with NCCs who were overly fixated with CareFirst program rules rather than prioritizing patient care.

Care Plans. For most providers, the individualized care plans were viewed positively as an effective method of communication between the NCC and physician regarding patient care management goals, patient needs, and resolving patient-related issues. Providers viewed care plans as particularly valuable for patients with complex needs - for example, diabetics were commonly cited in this context.

... patients that were in my care plans benefit, especially because they were diabetics and ... I was getting the data more

Although care plans served as a valuable care coordination tool, some providers remarked that care plans did not help with clinical decision-making. In this context, providers preferred to use their existing medical records.

... it coordinates the care that we need to delineate ... this gives us an idea or a goal of what to achieve. If we don't have some sort of a list ... then how are we going to move forward? I think the care plan provides us that ... does it help me as a clinician take care of my patient? I would say no. 
While care plans were valued as a way to facilitate care coordination and encourage patient engagement in better selfmanagement of conditions, a few providers expressed several concerns with the care plans. First, some patients were initially assessed a fee for care plan enrollment, which created a barrier to access for these patients. However, the care plan fees were later removed. Second, many physicians expressed uncertainty about care plan eligibility requirements and had differing views on which patients could benefit from the care plan process. Also, providers described difficulties in connecting some patients with mental health services and resources.

... I have some patients that need a care plan ... but when you talk about the money and how much they're going to be paying, they say, "Oh, this is so good but ouch, doctor.

Outcome Incentive Awards. Providers held mixed views on whether OIA bonuses influenced their motivation or engagement with the CareFirst PCMH program. Perceptions of OIAs varied across panels. For example, most providers in large health systems revealed that they did not receive or were unaware of how OIAs were distributed to individual providers. However, providers in small independent practices, where the OIA was a valuable revenue stream for practice operations, thought the OIA was key to engagement. In contrast, other providers thought the OIA was not influential - stating that the most valuable part of the program was the additional support of the NCC and other resources.

By all means, the outcome incentive award does not hurt, and it is always nice to be compensated for the extra time. I wouldn't say my participation is because of that. To me, the biggest value is having that additional care management support ... Several grievances were raised about the distribution of OIAs, which were related to fairness, distrust of data, and a lack of transparency on how OIAs were calculated. Many providers reported not receiving OIA bonuses when they claimed they earned it. Others said that rewarding groups based on improvement from their prior year's baseline performance was unfair for high performing practices. Additionally, a number of providers said the data and methods used to determine OIAs were not accurate or transparent.

It's been very hard for us. We were so good the year before we entered the program. The bar was so high that we've been unable to reach that bar any longer despite doing the best that we possibly could ... While CareFirst made multiple changes to the OIA criteria over the past few years and made extensive efforts to communicate the changes with clinicians, most providers remained confused about the criteria or distrustful of the data used to calculate the OIA, even though a majority of all participating providers earned an OIA based on other program data. ${ }^{16}$

Panel Meetings. Panel meetings were implemented after 2011 as a requirement for provider engagement, which was one of several performance measures used by CareFirst to calculate OIAs. Panel meetings were largely viewed as unproductive and inconvenient. Some providers felt that numbers were presented by CareFirst's program consultants without background context. Many providers were displeased when program consultants dismissed their concerns or questions about the data. Other providers thought the panel meetings created an unpleasant, competitive environment.

Yeah, the quarterly meetings are not helpful in that way, in improving anything. It's just more like data sharing.

What we say matters nothing. They have their rules. They have their guidelines. That's all that counts.

Searchlight Data Portal. During the initial years of the program, few providers used the Searchlight data portal. Over time, increased physician engagement with the data portal was facilitated by CareFirst representatives who helped with logging into Searchlight and printing out reports for clinicians and office staff. However, most providers viewed the Searchlight data portal as the least valuable program element. One reason for this view was the data portal was inconvenient and difficult to access. For example, the password requirements were overly stringent, and passwords expired on a frequent basis. In addition, providers did not find the content useful nor the interface user-friendly.

... it's tough to ask a provider in a seriously busy environment to $\log$ on to one more thing and look. Just somehow, this has to run where it's not creating a lot of extra work for us. That's just the bottom line, really.

\section{Provider Perspectives: Cross-Cutting Themes}

Some Providers at Odds with Top-Down Approach to Program Design. Some providers had a distrustful relationship with CareFirst throughout the PCMH initiative, while other physicians expressed a collaborative relationship. A number of providers felt excluded from the decision-making process and felt as if CareFirst employed a top-down approach to program design. This sentiment was expressed when providers discussed the quarterly panel meetings, program consultants, and CareFirst data reports. Some providers believed their feedback on program challenges, requirements, and expectations was ignored. Other providers felt their time and professional autonomy were devalued when they were 
expected to call high-cost specialists to discuss ways for specialists to be more cost-efficient. A suggestion by providers to mitigate this top-down approach was to create a board of physician representatives from each panel to review and discuss changes to program requirements with CareFirst leadership. Representative quotes for each of the cross-cutting themes are available in the online appendix.

Communication Affects Provider Engagement and Motivation. Some providers perceived a contributing factor to tension with CareFirst was limited two-way communication. Many participating providers were not aware of important program requirements and expectations. Specifically, participants described a lack of transparency on how OIAs were calculated, criteria used to rate high-cost specialists, and the selection of patients for care plans. Communication between CareFirst representatives and providers during panel meetings was viewed as punitive and one-sided. A few providers expressed skepticism of the data presented and found that CareFirst representatives were not responsive to their comments or questions. Some providers believed that communication challenges led to a strained relationship between providers and CareFirst, which negatively influenced their level of engagement with the program.

Differing Priorities on Selecting Patients for Care Plans. One key theme that emerged was differing priorities between providers and CareFirst on selecting patients for care plans. The CareFirst PCMH goal of selecting historically high-cost patients for care plans differed at times with provider instincts to prioritize high-risk patients to reduce future hospitalizations. Some providers wanted to enroll high-risk patients whom CareFirst found to be ineligible because they did not have high historical costs. A few clinicians described situations where high-risk patients did not appear on CareFirst's eligibility reports. The CareFirst PCMH program, which focused on making the best use of limited resources, sought to identify a select group of historically high-cost patients for care plans - an approach perceived as short-sighted by some providers who thought they could help improve patients' conditions and prevent or delay spiraling utilization down the road.

Some Providers Expressed Limited Trust in and Utility of CareFirst Data. Various cost-related data helped to inform decisions on selecting patients for care plans, specialist referrals, and prescribing medications. Providers were also given data reports on OIA bonuses and panel-wide statistics. However, some providers raised concerns about limited trust in the accuracy of CareFirst's data reports. Several issues were raised, including the misattribution of patients to a specific physician, the inclusion of deceased patients in reports, and a lack of timely data updates. Also, a few providers mentioned that CareFirst's reports had limited utility. First, some providers did not find panel aggregated data to be useful because it was not specific to an individual practice. Second, some providers refused to make cost-cutting decisions at the expense of quality. For example, PCPs said that they would not choose a specialist who is relatively low-cost over a trusted specialist who provided high-quality care. Third, providers expressed concern that data without context had limited utility. For example, some patients who had a single past episode of expensive care were grouped as "high-cost" patients eligible for care plans, even though their condition had stabilized.

PCMH Requirements Viewed as a Burden on Time and Competing Demands. Some providers expressed the view that the PCMH program requirements were extensive and demanding. For example, the requirement to $\log$ into the Searchlight data portal on a regular basis, attend mandatory panel meetings each quarter, review data reports from CareFirst, fill out care plan paperwork, and recruit patients to be eligible for care plans were viewed as additional burdens on providers because it took time away from patient care. In addition, a majority of providers made clear that they were pressed for time and overwhelmed by competing demands.

\section{DISCUSSION}

Our study findings revealed that provider experiences with individual PCMH program elements varied considerably. First, nurse care coordinators (NCCs) represented the single most valuable element of the program. Over time, NCCs became more integrated with practices as trusted care team members who assumed greater roles in patient care and PCMH administrative tasks. This finding is consistent with other studies that identify nurses as critical team members in enhancing care coordination. ${ }^{2,6}$ Second, individualized patient care plans were viewed favorably as a way to improve care coordination and communication with the NCCs. However, providers held mixed views on the outcome incentive awards (OIAs). These results are consistent with a prior study that found many physicians did not understand or care about the OIAs. ${ }^{14}$ Some providers did not find panel meetings to be a productive use of their time. Finally, a majority of providers viewed the Searchlight data portal as the least valuable element of the PCMH program.

We also identified several cross-cutting themes based on the perspectives of PCMH providers. First, some providers felt alienated from decisions on program design and perceived a top-down approach in how the PCMH program was being implemented. Second, communication with CareFirst influenced provider engagement. For some providers, strained relationships in panel meetings were due to perceptions that CareFirst representatives had been dismissive of comments and some questions. Third, some providers felt that CareFirst had a different priority for selecting high-cost patients for care plans based on historical data, instead of a proactive approach that sought to prevent high-risk patients from becoming highcost in the future. Fourth, a number of providers had limited trust in CareFirst's data reports. 
The implementation of a PCMH program can place additional time and administrative burden on primary care physicians who may already experience stress, dissatisfaction, and burnout. $^{20}$ One interpretation of this study's findings is that both the NCCs and individual care plans were highly valued because they improved care coordination for patients. In the CareFirst PCMH program, a key concern was limited opportunity for two-way communication between providers and CareFirst. Maintaining a high level of provider trust in payer-based reports is essential to provider engagement, buyin, and ownership of program results. Prior research has found that physician satisfaction is associated with a greater sense of control related to quality improvement activities. ${ }^{21}$ Our findings show that providers were concerned with PCMH program requirements, communication, data accuracy, and a perceived distance between CareFirst and themselves. Active provider engagement in the development of PCMH models may help to improve buy-in and decrease burden. ${ }^{22}$

\section{Limitations}

This study had several data limitations. First, the study sample for the qualitative analysis was limited to 65 participants in focus groups and 16 telephone interviews. Second, the analysis was restricted to providers within a single geographic region (Maryland and Northern Virginia), so these findings are not generalizable. Third, our findings may be subject to selection bias in that providers who agreed to participate in the focus groups may have differing views from those who declined to participate.

\section{CONCLUSION}

Results from this study have practical implications for the development of other payer-based PCMH initiatives. NCCs and the targeted use of individualized care plans for patients with complex needs are essential components in an effective PCMH program. Payers seek greater transformation in the delivery of primary care services with an increased focus on patient-centered, comprehensive, coordinated, high-quality, and efficient care. ${ }^{23}$ In 2019, as physician payment reforms take effect under the Medicare Access and CHIP Reauthorization Act (MACRA), growing interest in alternative payment models, which include advanced PCMH programs and accountable care organizations, will continue. ${ }^{24}$

Understanding provider perspectives and experiences with a payer-based $\mathrm{PCMH}$ program can guide the development and improvement of other payer-based initiatives. Our study, along with previous studies of the CareFirst PCMH program, provides contextual information on why specific program elements, such as the NCCs, are useful in improving care to patients and why other elements may be less useful, such as the Searchlight data portal. ${ }^{14,25}$ Successful implementation of a payer-based PCMH program depends on whether providers perceive the components of a program are useful for improving quality of care. Improving communication with providers and building trust in data reports are critical for effective implementation of a payer-based PCMH program. Future studies should examine the qualitative experiences of physicians in other payer-based PCMH programs and how these experiences affect provider engagement and satisfaction.

\section{Acknowledgments:}

We would like to thank Alan newman Research for their contributions in the production and gathering of written transcripts for this study.

Financial Disclosure: This program evaluation study was supported by a research grant from CareFirst BlueCross BlueShield of Maryland.

Corresponding Author: Gilbert Gimm, PhD; Department of Health Administration and Policy, George Mason University, Fairfax, VA, USA (e-mail:ggimm@gmu.edu).

\section{Compliance with Ethical Standards:}

All research protocols, focus group moderator guides and data collecition instruments were approved by the GMU Instituional Review Board. Each participant gave informed consent for the interview and audio-recording.

Conflict of Interest: Dr. Want is an employee of the Peterson Center on Healthcare and Want Healthcare LLC. Mr. Hough is an employee of Alan Newman Research. Dr. Nichols discloses receiving honoraria from the following organizations: WC Research, Inc.; Navigant Consulting; Institute for International Research; Kaufman and Conoles, P.C.; Conent, Inc.; and the Center for Corporate Innovation. All other authors declare they have no conflicts of interest.

\section{REFERENCES}

1. Patient-Centered Primary Care Collaborative (PCPCC). Joint principles of the patient-centered medical home, 2007. Available at: http://www. pcpcc.org/about/medical-home. Accessed on February 25, 2019.

2. O'Malley AS, Gourvetich $\mathbf{R}$, Draper $\mathbf{K}$, Bond A, Tirodkar MA. Overcoming challenges to teamwork in patient-centered medical homes: a qualitative study. J Gen Intern Med. 2015; 30(2): 183-192.

3. Friedman A, Howard J, Shaw E, Cohen D, Shahidi L, Ferrante J. Facilitator and barriers to care coordination in patient-centered medical homes (PCMHs) from coordinators' perspectives. J Am Board Fam Med. 2016; 29(1): 90-101.

4. Geonnotti K, Taylor E, Peikes D, et al. Engaging Primary Care Practices in Quality Improvement: Strategies for Practice Facilitators. AHRQ Publication No. 15-0015-EF. Rockville, MD: Agency for Healthcare Research and Quality: 2015.

5. Alexander JA, Markovitz AR, Paustian ML, et al. Implementation of patient-centered medical homes in adult primary care practices. Med Care Res Rev. 2015; 72(4): 438-467.

6. Gimm G, Want J, Hough D, Polk T, Rodan M, Nichols LM. Medical home implementation in small primary care practices: provider perspectives. J Am Board Fam Med. 2016; 29(1): 90-101.

7. Goldberg DG, Kuzel AJ. Elements of the patient-centered medical home in family practices in Virginia, Ann Fam Med. 2009; 7(4): 301-308.

8. Rittenhouse D, Casalino $\mathbf{L}$, Shortell $\mathbf{S}$, et al. Small and medium-size physician practices use few patient-centered medical home processes. Health Aff (Millwood). 2011; 30(8): 1575-1584.

9. Peikes D, Zutshi A, Genevro JL, Parchman ML, Meyers DS. Early evaluations of the medical home: building on a promising start. Am J Manag Care. 2012; 18(2):105-116.

10. Friedberg $\mathbf{M W}$, Schneider EC, Rosenthal MB, Volpp KG, Werner RM. Association between participation in a multipayer medical home intervention and changes in quality, utilization, and costs of care. JAMA. 2014; 311(8): 815-825 
11. Lemak CH, Nahra TA, Cohen GR, et al. Michigan's fee-for-value physician incentive program reduces spending and improves quality in primary care. Health Aff (Millwood). 2015; 34(4): 645-652.

12. Dale SB, Ghosh A, Peikes DN et al. Two-year costs and quality in the comprehensive primary care initiative. N Engl J Med. 2016; 374(24): 2345-2356.

13. Cuellar A, Helmchen LA, Gimm G, et al. The Carefirst patient-centered medical home program: cost and utilization effects in its first three years. J Gen Intern Med. 2016; 31(11):1382-1388

14. Afendulis CC, Hatfield LA, Landon BE, et al. Early impact of Carefirst's patient-centered medical home with strong financial incentives. Health Aff (Millwood). 2017; 36(3):468-475.

15. Marsteller JA, Hsu Y-J, Gill C, et al.Maryland multipayor patientcentered medical home program: a 4-year quasi-experimental evaluation of quality, utilization, patient satisfaction, and provider perceptions. Med Care. 2018; 56(4):308-320.

16. CareFirst Blue Cross Blue Shield. 2017 Program Guidelines and Description for CareFirst Patient-Centered Medical Home Program (PCMH). Available at: https://www.provider.carefirst.com/carefirst-resources/provider/pdf/pcmh-program-description-guidelines.pdf. Accessed on February 25, 2019.

17. Patton M. Qualitative evaluation and research methods. Beverly Hills, CA: Sage Publications; 1990:169-186.

18. Lincoln YS, Guba EG. Naturalistic Inquiry. Newbury Park, CA: Sage Publications; 1985:301-315.
19. Landis JR, Koch GG. The measurement of observer agreement for categorical data. Biometrics. 1977; 33: 159-174.

20. Richardson JE, Kern LM, Silver M, Jung HY, Kaushal R. Physician satisfaction in practices that transformed into patient-centered medical homes: a statewide study in New York. Am J Med Qual. 2016; 31(4): 331336.

21. Alidina S, Rosenthal MB, Schneider EC, Singer SJ, Friedberg MW. Practice environments and job satisfaction in patient-centered medical homes. Ann Fam Med. 2014; 12(4): 331-337.

22. West CP, Dyrbye LN, Rabatin JT, et al. Intervention to promote physician well-being, job satisfaction, and professionalism: a randomized clinical trial. JAMA Intern Med. 2014; 174(4):527-533

23. Shoemaker SJ, McNellis RJ, DeWalt DA. The capacity of primary care for improving evidence-based care: early findings from AHRQ's EvidenceNOW. Ann Fam Med. 2018; 16 (Suppl. 1): S2-S4.

24. Wilensky GR. Will MACRA improve physician reimbursement? N Engl J Med. 2018; 378 (14): 1269-1271

25. Peterson GG, Geonnotti KL, Hula L, et al. Association between extending Carefirst's medical home program to Medicare patients and quality of care, utilization, and spending. JAMA Intern Med. 2017;177(9): 1334-1342

Publisher's Note Springer Nature remains neutral with regard to jurisdictional claims in published maps and institutional affiliations. 